\title{
Global Dynamics of Infectious Disease with Arbitrary Distributed Infectious Period on Complex Networks
}

\author{
Xiaoguang Zhang, ${ }^{1,2}$ Rui Song, ${ }^{2}$ Gui-Quan Sun, ${ }^{2,3}$ and Zhen Jin ${ }^{2,3}$ \\ ${ }^{1}$ School of Mechatronic Engineering, North University of China, Taiyuan, Shanxi 030051, China \\ ${ }^{2}$ Department of Mathematics, North University of China, Taiyuan, Shanxi 030051, China \\ ${ }^{3}$ Complex Systems Research Center, Shanxi University, Taiyuan, Shanxi 030006, China
}

Correspondence should be addressed to Zhen Jin; jinzhn@263.net

Received 6 July 2014; Accepted 19 August 2014; Published 1 September 2014

Academic Editor: Sanling Yuan

Copyright (C) 2014 Xiaoguang Zhang et al. This is an open access article distributed under the Creative Commons Attribution License, which permits unrestricted use, distribution, and reproduction in any medium, provided the original work is properly cited.

\begin{abstract}
Most of the current epidemic models assume that the infectious period follows an exponential distribution. However, due to individual heterogeneity and epidemic diversity, these models fail to describe the distribution of infectious periods precisely. We establish a SIS epidemic model with multistaged progression of infectious periods on complex networks, which can be used to characterize arbitrary distributions of infectious periods of the individuals. By using mathematical analysis, the basic reproduction number $R_{0}$ for the model is derived. We verify that the $R_{0}$ depends on the average distributions of infection periods for different types of infective individuals, which extend the general theory obtained from the single infectious period epidemic models. It is proved that if $R_{0}<1$, then the disease-free equilibrium is globally asymptotically stable; otherwise the unique endemic equilibrium exists such that it is globally asymptotically attractive. Finally numerical simulations hold for the validity of our theoretical results is given.
\end{abstract}

\section{Introduction}

The infectious period of an infective individual means the period during which an infected person has a probability of transmitting the virus to any susceptible host or vector they contact. Note that the infectious period may be associated with the fitness of persons. The influence degrees of infection and rates of disease transmission are varied for individuals with different infectious periods. Every year, some emerging infectious diseases with unknown infectious period are seriously threatening the health of people. There is no doubt that the deficiency of the infectious period's knowledge results in the difficulty of controlling epidemic. Then, in order to obtain the date of the infectious period of these epidemics in medicine, a large amount of statistics data is necessary. However, it is hard to get the date in the early stage of the disease. Therefore applying mathematical methods to research the effects of infectious period distribution on the infectious diseases spread is significative.
As the SIS compartment model was first proposed by Kermack and McKendrick in 1932 [1], thousands of scientists successively started to study the epidemic propagation by mathematic models [2-4]. In most of their models, infected compartment contains all infected individuals and the proportion of infected individuals who transit into the next state per unit time is a constant $\gamma$. Wearing et al. [5] pointed out that the assumption of exponentially distributed infectious periods always results in underestimating the basic reproductive ratio of an infection from outbreak data. According to the staged progression features of HIV or TB, Lloyd [6] applied gamma distribution to describe the infectious period distribution. However, the distributions of the infectious period of a lot of infectious diseases in the real world may not satisfy exponent or gamma distribution. Then, Feng et al. [7] used integral-differential equations to study the nonexponential distribution of the infectious period. The homogeneous mixing models, they considered, ignore the heterogeneity of contacts of individuals. 
The network origins from the well-known six degrees of separation theory. The small-world (SW) property is the most popular feature in complex network theory [8]. The SW networks constitute a mathematical model for social networks that show two types. The first type can be called exponential networks since there is the probability of finding a node with degree $k$ different from the average degree $\langle k\rangle$ which decays exponentially fast for large $k$. The second kind of networks comprises those referred to as scale-free (SF) networks. For these networks, the probability that a given node is connected to $k$ other nodes follows a power law of the form $P(k) \sim k^{-v}$, with the remarkable feature that $2<v \leqslant 3$ for most real world networks.

With the development of networks research, there have been some researchers studying infectious disease models on networks for decades, such as SIS model proposed by PastorSatorras and Vespignani in 2001 [9], SIR model established by Yang et al. in 2007 [10], and SI pattern model introduced by Barthélemy et al. in 2004 [11]. Hence studying the epidemic model with infectious period distribution on networks is necessary and meaningful. However, there is little literature about the infectious period distribution problems based on networks. Zhang et al. in 2011 proposed a susceptibleinfected-susceptible staged progression and different infectivity models on different complex networks [12], where the infectious period follows a gamma distribution. Zager and Verghese in 2009 established a discrete differential equation to present an arbitrarily distributed infectious period [13]. Moreover, they studied epidemic thresholds for infections on uncertain networks. However, the former researchers did not analyze the stability of equilibrium theoretically. Therefore, we build continuous-time ordinary differential equations to study an arbitrarily distributed infectious period epidemic model on networks. We extend the scope of previous works in this area to include dynamic analysis results mathematically.

The rest of the paper is organized as follows. In Section 2, we establish an SIS model with an arbitrary distribution of infectious period on complex networks. We compute the basic reproduction number and analyze the globally asymptotic stability of the disease-free equilibrium and the global dynamics of the endemic equilibrium in Section 3. In Section 4, we perform numerical simulations to verify the above theories. Finally, a brief conclusion and discussion will be given in Section 5 .

\section{The Model}

One feature of some diseases is that different patients may have different symptoms. The feature had appeared in some patients of some SIR/SI diseases, such as TB/HIV studied by Guo and Li in 2006 [14]. However, the other feature that different groups may have different infected stage processes is ignored. We propose a modified staged progression model to capture the second feature above. In our model, the infectious period distributions of different groups follow different gamma distributions. The linear combination of different gamma distributions can be transformed into normal distribution, chi-square distribution, exponential distribution,
Erlang distribution, or beta distribution [15]. So our model can be transformed into any distributions.

We classify the population as infected $I_{(i, j)}$ and susceptible $S$. Compartment $I_{(i, j)}$ contains those individuals whose infectious period has $i$ stages and which are now in the $j$ th stage $(j \leqslant i)$. Susceptible individuals enter into the first stage $I_{(i, 1)}$ after being infected and then gradually progress from this stage to stage $I_{(i, M)}(i=1,2, \ldots n, M \leqslant i)$. The infected individuals in the $I_{(i, M)}$ stage will be susceptible again.

In contrast to classical compartment models, we consider the whole population and their contacts on networks. Each individual in the community can be regarded as a vertex in the network, and each contact between two individuals is represented as an edge (line) connecting these vertices. The number of edges emanating from a vertex, that is, the number of contacts a person has, is called the degree of the vertex. Therefore, we assume that the population is divided into $n$ distinct groups of sizes $N_{k}(k=1,2, \ldots, n \rightarrow \infty)$ such that each individual in group $k$ has exactly $k$ contacts per day. If the whole population size is $N\left(N=N_{1}+N_{2}+\cdots+\right.$ $N_{n}$ ), then the probability that a uniformly chosen individual has $k$ contacts is $P(k)=N_{k} / N$, which is called the degree distribution of the network. The average degree is $\langle k\rangle=$ $\sum_{k=1}^{n} k P(k)$.

Let $S_{k}(t)$ denote the number of susceptible nodes of degree $k$ at time $t$. Let $I_{k(i, j)}(t)$ be the number of infected nodes whose infectious period has $i$ stages and which are now in the $j$ th stage of their infection $(j \leqslant i)$ of degree $k$ at time $t$. The transmission sketch is shown in Figure 1.

We make the following basic assumptions about the infectious disease models.

(1) Here, we will not incorporate the possibility of individual removal due to birth and death or acquired immunization. That is to say, the total population $N(t)=\sum_{k=1}^{n}\left(S_{k}(t)+\sum_{i=1}^{M} \sum_{j=1}^{i} I_{k(i, j)}(t)\right)$ is a constant.

(2) The stages in infectious period can be given by the discrete random variable $X$. The range of values of $X$ need not to be finite, but for ease of presentation we assume that $X$ can only take values from 1 to $M$. The probability of infected individuals with an infectious period of exactly $i$ stages is $q_{i}$.

(3) The mutants of virus can cause the same individual to suffer different infected stage progression processes if he or she is infected again.

(4) In order to analyze the model simply and efficiently, all transmission rates from infected individuals to susceptible individuals are $\beta$. The duration of infected individuals $I_{k(i, j)}$ in compartment $(i, j)$ th is $1 / \gamma$.

(5) In a network with no assortative (i.e., disassortative) then the conditional probability $P\left(k^{\prime} \mid k\right)$ that a given vertex with degree $k$ is linked to a vertex with degree $k^{\prime}$ by one edge is proportional to $k P(k)$, which is independent of its own vertex degree $k$ [9], and hence we have $P\left(k^{\prime} \mid k\right)=k^{\prime} P\left(k^{\prime}\right) /\langle k\rangle$. The expectation that any given edge points to an infected vertex becomes $\Theta=\sum_{k=1}^{n} \sum_{i=1}^{M} \sum_{j=1}^{i} k I_{k(i, j)} / \sum_{k=1}^{n} k N_{k}$. 


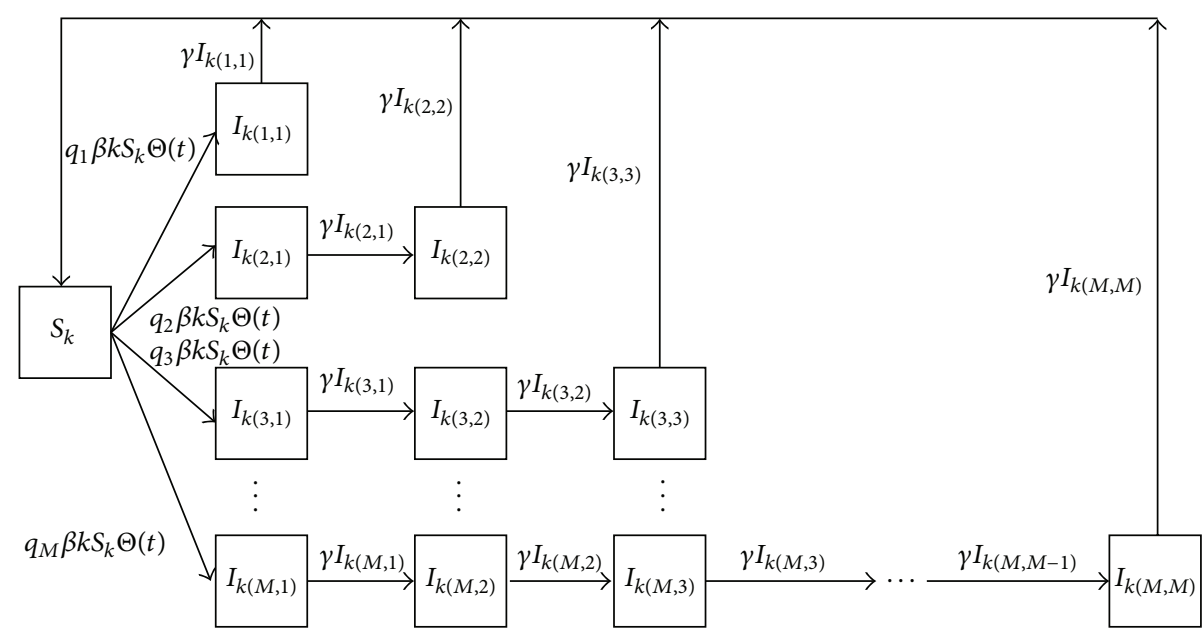

FIGURE 1: The flowchart of disease spreading.

On the basis of the above assumption, the model of $n(M(M+1) / 2+1)$ ordinary differential equations with $n(n \geqslant$ $1)$ the maximum degree and $M(M \geqslant 1)$ the maximum infection stages in infectious period is as follows:

$$
\begin{aligned}
\frac{d S_{k}(t)}{d t} & =-\beta k S_{k}(t) \Theta+\gamma \sum_{i=1}^{M} I_{k(i, i)}(t), \\
\frac{d I_{k(i, 1)}(t)}{d t} & =q_{i} \beta k S_{k}(t) \Theta-\gamma I_{k(i, 1)}(t), \\
\frac{d I_{k(i, j)}(t)}{d t} & =\gamma I_{k(i, j-1)}(t)-\gamma I_{k(i, j)}(t),
\end{aligned}
$$

where $k=1, \ldots, n, i=1, \ldots, M$, and $2 \leqslant j \leqslant i$. For system (1), since the total population is constant, we can only consider the infected compartments below. For a given degree distribution $P(k)$, the number of the nodes which have the same degree $k, N_{k}=S_{k}+I_{k}=P(k) N$, is definite, where $I_{k}=\sum_{i=1}^{M} \sum_{j=1}^{i} I_{k(i, j)}$.

The relative densities of susceptible and infected nodes with an infectious period of $i$ stages and which are now in the $j$ th stage of degree $k$ at time $t$ are denoted by $s_{k}(t)=$ $S_{k}(t) / N_{k}(t)$ and $\rho_{k(i, j)}(t)=I_{k(i, j)}(t) / N_{k}(t)$, respectively. Without loss of generality, we set $\gamma=1$, since it only affects the definition of the time scale of the epidemic transmission.

System (1) can be rewritten as

$$
\begin{gathered}
\frac{d \rho_{k(i, 1)}(t)}{d t}=q_{i} \beta k\left(1-\phi_{k}(t)\right) \Theta-\rho_{k(i, 1)}(t), \\
\frac{d \rho_{k(i, j)}(t)}{d t}=\rho_{k(i, j-1)}(t)-\rho_{k(i, j)}(t),
\end{gathered}
$$

where $k=1, \ldots, n, i=1, \ldots, M$, and $2 \leqslant j \leqslant i$, and $\phi_{k}(t)=\sum_{i=1}^{M} \sum_{j=1}^{i} \rho_{k(i, j)}(t)$ denotes the density of infected nodes of degree $k$ at time $t$. Therefore the average infectious period $\langle i\rangle$ is $\sum_{i=1}^{M} i q_{i}$. Then one can derive that $\Theta=$ $(1 /\langle k\rangle) \sum_{k=1}^{n} k P(k) \phi_{k}(t)$.

\section{The Analysis of Model (2)}

3.1. Basic Reproduction Number. We will compute the basic reproduction number using the next-generation matrix proposed by van den Driessche and Watmough [16]. For convenience we define $M(M+1) / 2=\mathrm{M}$ and $n(M(M+1) / 2)=\mathrm{N}$. It is easy to verify that system (2) has a unique diseasefree equilibrium $E_{0}=\overbrace{(0,0, \ldots, 0)}^{N}$. We note that only compartments $\rho_{k(i, j)}$ are involved in the calculation of $R_{0}$. In the disease-free state $E_{0}$, the rate of appearance of new infections $F$ and the rate of transfer of individuals out of the compartments $V$ are given by

$$
F=\frac{\beta}{\langle k\rangle}\left[\begin{array}{ccccc}
F_{11} & F_{12} & F_{13} & \cdots & F_{1 n} \\
F_{21} & F_{22} & F_{23} & \cdots & F_{2 n} \\
F_{31} & F_{32} & F_{33} & \cdots & F_{3 n} \\
\vdots & \vdots & \vdots & \cdots & \vdots \\
F_{n 1} & F_{n 2} & F_{n 3} & \cdots & F_{n n}
\end{array}\right]_{\mathrm{N} \times \mathrm{N}}
$$

where $F_{i j}(1 \leqslant i \leqslant n, 1 \leqslant j \leqslant n)$ are the $\mathrm{M} \times \mathrm{M}$ matrices

$$
F_{i j}=i j p(j)\left[\begin{array}{c}
A_{1} \\
A_{2} \\
\vdots \\
A_{M}
\end{array}\right]_{\mathrm{M} \times \mathrm{M}}
$$

where

$$
\begin{gathered}
A_{l}=\left[\begin{array}{cccc}
q_{l} & q_{l} & \cdots & q_{l} \\
0 & 0 & \cdots & 0 \\
\vdots & \vdots & \cdots & \vdots \\
0 & 0 & \cdots & 0
\end{array}\right]_{l \times \mathrm{M}}, \quad 1 \leqslant l \leqslant M, \\
V=\left[\begin{array}{llll}
V_{*} & & & \\
& V_{*} & & \\
& & \ddots & \\
& & & V_{*}
\end{array}\right]_{\mathrm{N} \times \mathrm{N}},
\end{gathered}
$$


where $V_{*}$ is the $\mathrm{M} \times \mathrm{M}$ matrices

$$
V_{*}=\left[\begin{array}{llll}
V_{* 1} & & & \\
& V_{* 2} & & \\
& & \ddots & \\
& & & V_{* M}
\end{array}\right]_{\mathrm{M} \times \mathrm{M}},
$$

in which $V_{*}$ is a block diagonal matrix with $M$ blocks and $V_{* i}$ are $i \times i$ matrices with entries of 1 on the diagonal and -1 on the first subdiagonal.

Using the concepts of next-generation matrix [16], the reproduction number is given by $R_{0}=\rho\left(F V^{-1}\right)$, that is, the spectral radius of the matrix $F V^{-1}$. We first represent the inverse of $V$ by the following matrix:

$$
V^{-1}=\left[\begin{array}{llll}
V_{*}^{-1} & & & \\
& V_{*}^{-1} & & \\
& & \ddots & \\
& & & V_{*}^{-1}
\end{array}\right]_{\mathrm{N} \times \mathrm{N}},
$$

and since $V_{*}$ is block diagonal with blocks $V_{* i}$, its inverse will be block diagonal with blocks $V_{* i}^{-1}$ which is $i \times i$ lower triangular matrices with entries of 1 .

Setting $C=F V^{-1}$, we have

$$
C=F V^{-1}=\left[C_{i j}\right]_{\mathrm{N} \times \mathrm{N}^{\prime}}
$$

where $C_{i j}(1 \leqslant i \leqslant n, 1 \leqslant j \leqslant n)$ are $\mathrm{M} \times \mathrm{M}$ matrices and

$$
C_{i j}=i j p(j)\left[\begin{array}{c}
B_{1} \\
\vdots \\
B_{M}
\end{array}\right],
$$

where

$$
B_{l}=\left[\begin{array}{c}
q_{l} D_{l} \\
0 \\
0 \\
\vdots \\
0
\end{array}\right]_{l \times M}, \quad 1 \leqslant l \leqslant M
$$

where $D_{l}=(M, M-1, M-2, \ldots, 1)_{1 \times \mathrm{M}}$.

Now we are ready to compute the eigenvalues of the matrix $C=F V^{-1}$. Thus, we obtain that the basic reproduction number $R_{0}$ which is the largest modulus of the roots of the characteristic equation below

$$
|\lambda E-C|=0
$$

To simplify and compute (11), we have

$$
\lambda^{\mathrm{N}-1}\left(\lambda-\beta \sum_{i=1}^{M} i q_{i} \frac{\left\langle k^{2}\right\rangle}{\langle k\rangle}\right)=0 .
$$

Therefore, we obtain the reproduction number

$$
R_{0}=\beta\langle i\rangle \frac{\left\langle k^{2}\right\rangle}{\langle k\rangle} .
$$

If $R_{0}>1$, we can derive the critical transmission rate from (13), which is

$$
\beta\langle i\rangle>\frac{\langle k\rangle}{\left\langle k^{2}\right\rangle} .
$$

Because the degree distribution of scale-free network is $P(k) \sim k^{-v}$, with $2<v \leqslant 3$ in most cases, for which $\left\langle k^{2}\right\rangle \rightarrow+\infty$, when the size of network is sufficiently large, then inequality (14) is always satisfied. In other words, the multistaged progression model will prevail on sufficiently large heterogenous networks more easily.

3.2. Global Stability of Disease-Free Equilibrium. In order to study the global stability of the disease-free equilibrium $E_{0}$, we first give the following lemma, which guarantees that the densities of each infected class cannot become negative and the sum of the densities of infective individuals with the same degree cannot be greater than unity.

Let $\rho_{k(i, j)}(t)=y_{(k, i, j)}(t)(k=1, \ldots, n, i=1, \ldots, M, j=$ $1, \ldots, i)$. Since $\phi_{k}=\sum_{i=1}^{M} \sum_{j=1}^{i} I_{k(i, j)}(t) / N_{k}(t) \in[0,1]$, we study system (2) for $\left(\sum_{i=1}^{M} \sum_{j=i}^{i} y_{(1, i, j)}, \sum_{i=1}^{M} \sum_{j=i}^{i} y_{(2, i, j)}, \ldots\right.$, $\left.\sum_{i=1}^{M} \sum_{j=i}^{i} y_{(n, i, j)}\right) \in \Delta_{\mathrm{N}}=\prod_{l=1}^{n}[0,1]$.

Lemma 1 (see [17]). The set $\Delta_{\mathrm{N}}$ is positively invariant for the system (2).

Proof. We will show that if $y(0) \in \Delta_{\mathrm{N}}$, then $y(t) \in \Delta_{\mathrm{N}}$ for all $t>0$. Denote

$$
\begin{aligned}
& \partial \Delta_{\mathrm{N}}^{1}=\left\{y \in \Delta_{\mathrm{N}} \mid \sum_{i=1}^{M} \sum_{j=1}^{i} y_{(l, i, j)}=0 \text { for some } 1\right\}, \\
& \partial \Delta_{\mathrm{N}}^{2}=\left\{y \in \Delta_{\mathrm{N}} \mid \sum_{i=1}^{M} \sum_{j=1}^{i} y_{(l, i, j)}=1 \text { for some } 1\right\} .
\end{aligned}
$$

Let the outer normals be denoted by $\eta_{l}^{1}=(\overbrace{0, \ldots,-1}^{l \text { th }}, \ldots, 0)$ and $\eta_{l}^{2}=(\overbrace{0, \ldots,+1}^{l \text { th }}, \ldots, 0)$.

For arbitrary compact set $\Omega$, Yorke had proved that $\Omega$ is invariant for $d x / d t=f(x)$, if, at each point $y$ in $\partial \Omega$ (the boundary of $\Omega$ ), the vector $f(y)$ is tangent or pointing to the set [18]. We can easily apply the result here, since $\Omega$ is an $n$-dimensional rectangle. Through Yorke's result, it is not difficult to obtain that

$$
\begin{aligned}
& \left(\left.\frac{d y}{d t}\right|_{\sum_{i=1}^{M} \sum_{j=1}^{i} y_{(l, i, j)=0}} \cdot \eta_{l}^{1}\right) \\
& \quad=-\left(\frac{q_{i} \beta l}{\langle k\rangle} \sum_{k \neq l} k P(k) \sum_{i=1}^{M} \sum_{j=1}^{i} y_{(k, i, j)}\right) \leqslant 0, \quad l=1, \ldots, n,
\end{aligned}
$$

$$
\left(\left.\frac{d y}{d t}\right|_{\sum_{i=1}^{M} \sum_{j=1}^{i} y_{(l, i, j)=1}} \cdot \eta_{l}^{2}\right) \leqslant 0, \quad l=1, \ldots, n .
$$


Hence, any solution that starts in $y \in \partial \Delta_{\mathrm{N}}^{1} \cup \partial \Delta_{\mathrm{N}}^{2}$ stays inside $\Delta_{\mathrm{N}}$.

By letting $y=\left(y_{1}^{\prime}, y_{2}^{\prime}, \ldots, y_{n}^{\prime}\right)^{T}$, where $y_{l}^{\prime}=\left(y_{(l, 1,1)}, y_{(l, 2,1)}\right.$, $\left.y_{(l, 2,2)}, \ldots, y_{(l, M, 1)}, \ldots, y_{(l, M, M)}\right)^{T}(1 \leqslant l \leqslant n)$ and $H(y)=$ $\left(H_{1}, H_{2}, \ldots, H_{n}\right)^{T}$, in which $H_{l}$ is a column vector with $\mathrm{M}$ rows and $H_{l}=-\left(q_{1} l \psi_{l} \Theta(y(t)), q_{2} l \psi_{l} \Theta(y(t)), y_{(l, 2,1)}, \ldots\right.$, $\left.q_{M} l \psi_{l} \Theta(y(t)), y_{(l, M, 1)}, \ldots, y_{(l, M, M-1)}\right)^{T}$, and $\psi_{l}=\sum_{i=1}^{M} \sum_{j=1}^{i}$ $y_{(l, i, j)}, \Theta(y(t))=(1 /\langle k\rangle) \sum_{l=1}^{n} l p(l) \sum_{i=1}^{M} \sum_{j=1}^{i} y_{(l, i, j)} \geqslant 0$. In the following, we use the method introduced in [19] to demonstrate the global behavior of the system (2). Then, (2) can be rewritten as a compact vector form

$$
\frac{d y}{d t}=A y+H(y)
$$

where $A y$ is the linear part of $y, H(y) \leqslant 0$ is the nonlinear part of $y$, and $A=F-V$.

Denote $s(A)=\max _{1 \leqslant l \leqslant n} \operatorname{Re}\left(\lambda_{l}\right)$, where $\lambda_{l}$ for $l=1, \ldots, \mathrm{N}$ are the eigenvalues of $A$, and Re represents the real part of the eigenvalues.

Remark 2. Consider $s(A)<0 \Leftrightarrow R_{0}<1 ; s(A)>0 \Leftrightarrow R_{0}>$ 1.

To obtain the global stability of the disease-free equilibrium $E_{0}$, we need the following lemma.

Lemma 3 (see [20]). Consider the system

$$
\frac{d y}{d t}=A y+H(y)
$$

where $A$ is an $n \times n$ matrix and $H(y)$ is continuously differentiable in a region $D \in R^{n}$. Assume that

(1) the compact convex set $C \subset D$ is positively invariant with respect to the system (18), and $\mathbf{0} \in C$;

(2) $\lim _{y \rightarrow 0}\|H(y)\| /\|y\|=0$;

(3) there exist $r>0$ and a (real) eigenvector $\omega$ of $A^{T}$ such that $(\omega \cdot y) \geqslant r\|y\|$ for all $y \in C$;

(4) $(\omega \cdot H(y)) \leqslant 0$ for all $y \in C$;

(5) $y=\mathbf{0}$ is the largest positively invariant set (for (18)) contained in $G=\{y \in C \mid(\omega \cdot H(y))=0\}$.

Then either $y=\mathbf{0}$ is globally asymptotically stable in $C$ or for any $y_{0} \in C \backslash\{\mathbf{0}\}$ the solution $\phi\left(t, y_{0}\right)$ of (18) satisfies $\liminf _{t \rightarrow \infty}\left\|\phi\left(t, y_{0}\right)\right\| \geqslant m$, where $m>0$, independent of the initial value $y_{0}$. Moreover, there exists a constant solution of (18), $y=y^{*}, y^{*} \in C \backslash\{\mathbf{0}\}$.

We will confirm that system (17) satisfies all the hypotheses of Lemma 3. Condition (1) of Lemma 3 is satisfied by letting $C=\Delta_{\mathrm{N}}$. For condition (3), notice that $A^{T}$ is irreducible and $a_{i j} \geqslant 0$ whenever $i \neq j$, and then there exists an eigenvector $\omega=\left(\omega_{(l, 1,1)}, \omega_{(l, 2,1)}, \omega_{(l, 2,2)}, \ldots, \omega_{(l, M, 1)}\right.$, $\left.\ldots, \omega_{(l, M, M)}\right)$ of $A^{T}$ and the associated eigenvalue is $s\left(A^{T}\right)$. If we let $\omega_{0}=\min _{l} \omega_{(l, i, j)}>0$, for $y \in \Delta_{\mathrm{N}}$, we then obtain

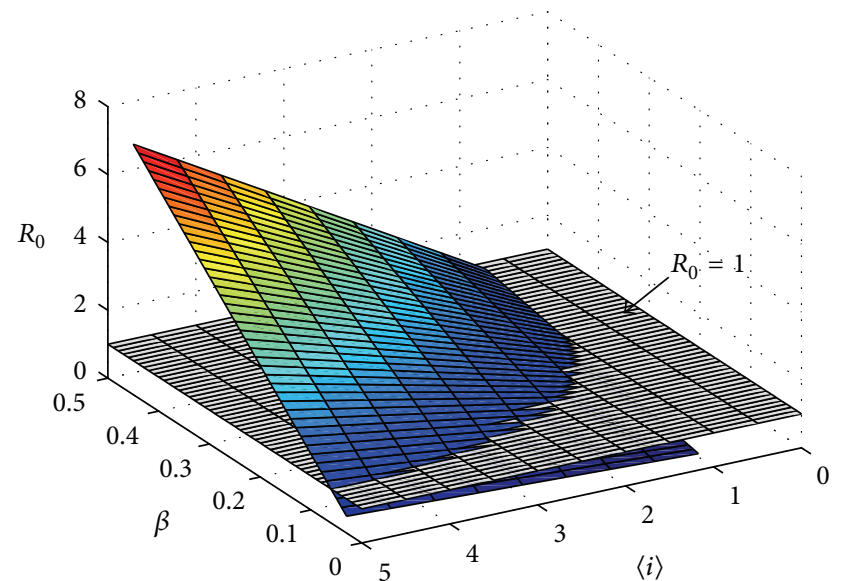

FIgURE 2: $R_{0}$ as a function of $\beta$ and $\langle i\rangle$, which depends on the heterogeneity of the social networks and the diversity of the infectious periods of the individuals.

$(\omega \cdot y) \geqslant \omega_{0} \sum_{k=1}^{n} \sum_{i=1}^{M} \sum_{j=1}^{i} y_{(k, i, j)} \geqslant \omega_{0}\left(\sum_{k=1}^{n} \sum_{i=1}^{M} \sum_{j=1}^{i}\right.$ $\left.y_{(k, i, j)}^{2}\right)^{1 / 2}$. Therefore $(\omega \cdot y) \geqslant r\|y\|$ for all $y \in \Delta_{N}$, where we set $r=\omega_{0}$. Conditions (2) and (4) are clearly satisfied.

To verify (5), we set $G=\left\{y \in \Delta_{\mathrm{N}} \mid(\omega \cdot H(y)=0)\right\}$. If $y \in G$, then $\sum_{l=1}^{n} \sum_{i=1}^{M} q_{i} l \psi_{l} \Theta(y(t)) \omega_{(l, i, 1)}+\sum_{l=1}^{n} \sum_{i=1}^{M} \sum_{j=2}^{i}$ $y_{(l, i, j)} \omega_{(l, i, j)}=0$. But since each term of the sum is nonnegative, $q_{i} l \psi_{l} \Theta(y(t)) \omega_{(l, i, 1)}=0$ for $l=1, \ldots, n, i=1, \ldots, M$ and $y_{(l, i, j)} \omega_{(l, i, j)}=0$ for $l=1, \ldots, n, i=1, \ldots, M, j=2, \ldots, i$, and then we have $y=\mathbf{0}$. Hence if $y \in G$, then $y=\mathbf{0}$. Therefore, the only invariant set with respect to (17) contained in $G$ is $y=\mathbf{0}$, and so condition (5) is satisfied.

Hence all the hypotheses of Lemma 3 are satisfied. Then either $R_{0}<1$ (the solution $y=\mathbf{0}$ is globally asymptotically stable in $\left.\Delta_{\mathrm{N}}\right)$ or $R_{0}>1$, and there exists a constant solution of (17), $y=y^{*}, y^{*} \in \Delta_{\mathrm{N}} \backslash\{\mathbf{0}\}$.

Theorem 4. If $R_{0}<1$, then the solution $y=\mathbf{0}$ (i.e., diseasefree equilibrium $E_{0}$ ) of the system (17) is globally asymptotically stable in $\Delta_{N}$; otherwise $R_{0}>1$, and there exists a constant solution $y^{*} \in \Delta_{\mathrm{N}} \backslash\{\mathbf{0}\}$.

3.3. Global Attractivity of Endemic Equilibrium. In the following, we will compute the value of the unique endemic equilibrium $E^{*}$ of the system (1), and we will use the method in [17] to ascertain global attractivity of the nonzero solution (i.e., endemic equilibrium).

For system (1), if $R_{0}>1$, we can derive the endemic equilibrium $E^{*}=(\overbrace{S_{1}^{*}, \ldots, S_{n}^{*}}^{n}, \overbrace{I_{1(1,1)}^{*}, \ldots, I_{1(M, M)}^{*}}^{M}, \ldots$, $\overbrace{\left.I_{n(1,1)}^{*}, \ldots, I_{n(M, M)}^{*}\right)}^{M}$, where $S_{k}^{*}=P(k) S, I_{k(i, 1)}^{*}=\left(q_{i} k \beta /\langle k\rangle\right) S_{k}^{*}$. $I^{\Delta}$, and $I_{k(i, j)}^{*}=I_{k(i, 1)}^{*}$, for $k=1, \ldots, n, i=1, \ldots, M$, $j=2, \ldots, i$, in which $S=\mathscr{K} / R_{0}, I^{\Delta}=\sum_{k=1}^{n} k P(k) I$, and $\mathscr{K}$ is the total number of nodes and keeps a constant, $I=\left(\left(R_{0}-1\right) / R_{0}\right) \mathscr{K}$. Then we can give the following theorem. 


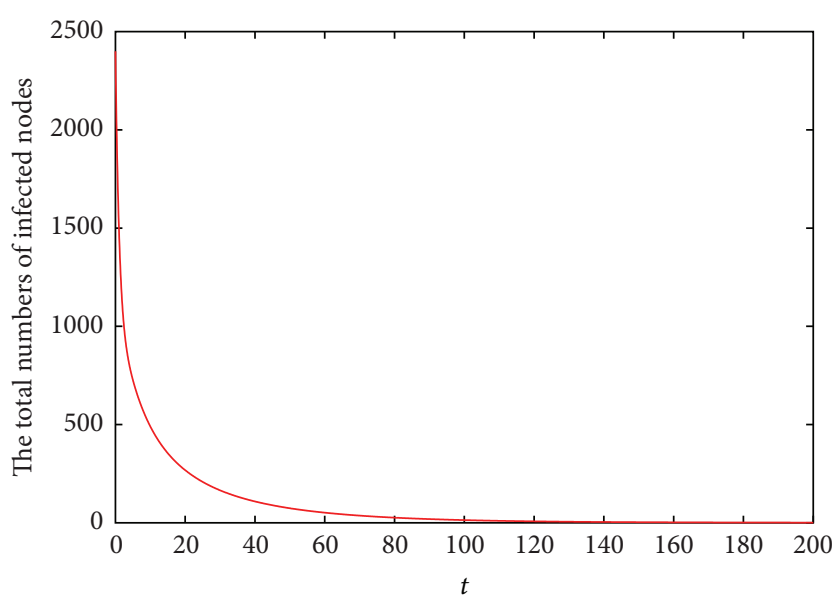

(a)

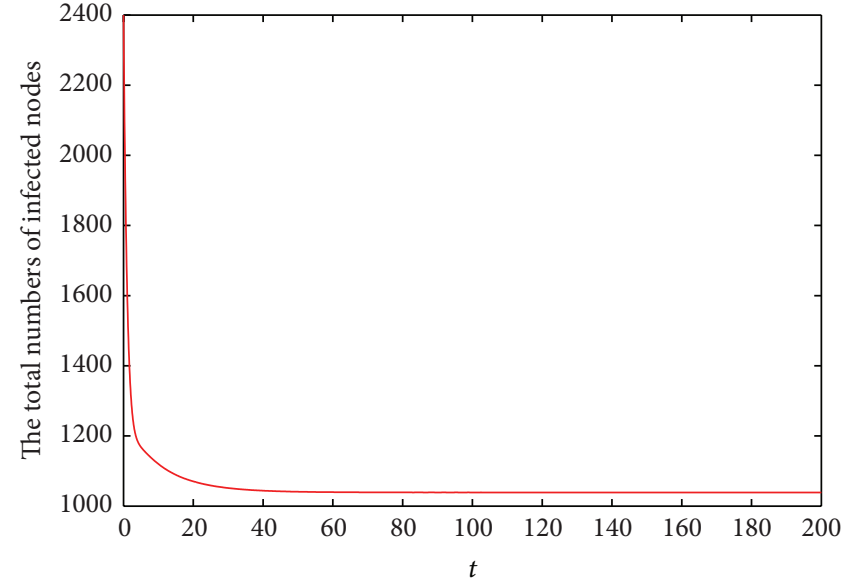

(b)

FIGURE 3: The total number of infected nodes on BA networks. We set parameters $M=4, q_{1}=0.1, q_{2}=0.2, q_{3}=0.3$, and $q_{4}=0.4$; that is, $\langle i\rangle=3$ and $\beta=0.037$ or $\beta=0.047$, which corresponds to $R_{0}=0.93<1((\mathrm{a}))$ or $R_{0}=1.18>1((\mathrm{~b}))$, respectively.

Theorem 5. If $R_{0}>1$, there exists a unique endemic solution of $(17) E^{*}=\left(S_{1}^{*}, \ldots, S_{n}^{*}, I_{1(1,1)}^{*}, \ldots, I_{1(M, M)}^{*}, \ldots, I_{n(1,1)}^{*}\right.$, $\left.\ldots, I_{n(M, M)}^{*}\right)$ such that $E^{*}$ is globally attractive in $\Delta_{\mathrm{N}} \backslash\{\mathbf{0}\}$.

Proof. We will prove that $y=\left(y_{1}^{*}, y_{2}^{*}, \ldots, y_{n}^{*}\right)^{T}$ is globally attractive in $\Delta_{\mathrm{N}} \backslash\{\mathbf{0}\}$, where $y_{l}^{*}=\left(y_{(l, 1,1)}^{*}, y_{(l, 2,1)}^{*}, y_{(l, 2,2)}^{*}, \ldots\right.$, $\left.y_{(l, M, 1)}^{*}, \ldots, y_{(l, M, M)}^{*}\right)$, and $y_{(l, i, j)}^{*}=I_{l(i, j)}^{*} / \sum_{i=1}^{M} \sum_{j=1}^{i} I_{l(i, j)}^{*}$. We define the following functions: $F: \Delta_{N} \rightarrow R$ and $f: \Delta_{\mathrm{N}} \rightarrow R$ for $y \in \Delta_{\mathrm{N}}$, where $F(y)=\max _{l}\left(y_{(l, i, j)} /\right.$ $\left.y_{(l, i, j)}^{*}\right), f(y)=\min _{l}\left(y_{(l, i, j)} / y_{(1, i, j)}^{*}\right) . F(y)$ and $f(y)$ are continuous and right-hand derivative exists along solutions of (17). We let $\psi_{l}^{*}=\sum_{i=1}^{M} \sum_{j=1}^{i} y_{(l, i, j)}^{*}$ and $y=y(t)$ be a solution of (17), and we may assume that $F(y(t))=y_{\left(l_{0}, i_{0}, j_{0}\right)}(t) / y_{\left(l_{0}, i_{0}, j_{0}\right)}^{*}$, $l=1, \ldots, n, i=1, \ldots, M, j=2, \ldots, i, t \in\left[t_{0}, t_{0}+\epsilon\right]$, for a given $t_{0}$ and for sufficiently small $\epsilon>0$. Then,

$$
\left.F^{\prime}\right|_{(17)}\left(y\left(t_{0}\right)\right)=\frac{y_{\left(l_{0}, i_{0}, j_{0}\right)}^{\prime}\left(t_{0}\right)}{y_{\left(l_{0}, i_{0}, j_{0}\right)}^{*}}, \quad t \in\left[t_{0}, t_{0}+\epsilon\right]
$$

where $\left.F^{\prime}\right|_{(17)}$ is defined as $\left.F^{\prime}\right|_{(17)}=\lim _{h \rightarrow 0^{+}} \sup ((F(y(t+h))-$ $F(y(t))) / h$ ). If $j_{0}=1$, from (17) we have

$$
\begin{aligned}
& y_{\left(l_{0}, i_{0}, 1\right)}^{*} \frac{y_{\left(l_{0}, i_{0}, 1\right)}^{\prime}\left(t_{0}\right)}{y_{\left(l_{0}, i_{0}, 1\right)}\left(t_{0}\right)} \\
& \quad=\left[1-\psi_{l_{0}}\right] q_{i} \beta l_{0} \Theta\left(y\left(t_{0}\right)\right) \frac{y_{\left(l_{0}, i_{0}, 1\right)}^{*}}{y_{\left(l_{0}, i_{0}, 1\right)}\left(t_{0}\right)}-y_{\left(l_{0}, i_{0}, 1\right)}^{*},
\end{aligned}
$$

or if $j_{0} \neq 1$, we have

$$
\begin{aligned}
y_{\left(l_{0}, i_{0}, j_{0}\right)}^{*} & \frac{y_{\left(l_{0}, i_{0}, j_{0}\right)}^{\prime}\left(t_{0}\right)}{y_{\left(l_{0}, i_{0}, j_{0}\right)}\left(t_{0}\right)} \\
=-y_{\left(l_{0}, i_{0},(j-1)_{0}\right)} & \frac{y_{\left(l_{0}, i_{0}, j_{0}\right)}^{*}}{y_{\left(l_{0}, i_{0}, j_{0}\right)}\left(t_{0}\right)}+y_{\left(l_{0}, i_{0}, j_{0}\right)}^{*} .
\end{aligned}
$$

According to the definition of $F(y(t))$, we have

$$
\frac{y_{\left(l_{0}, i_{0}, j_{0}\right)}\left(t_{0}\right)}{y_{\left(l_{0}, i_{0}, j_{0}\right)}^{*}} \geqslant \frac{y_{(l, i, j)}\left(t_{0}\right)}{y_{(l, i, j)}^{*}}, \quad 1 \leqslant l \leqslant n, 1 \leqslant i \leqslant M, 1 \leqslant j \leqslant i .
$$

Then if $F(y(t))>1$, we obtain

$$
y_{\left(l_{0}, i_{0}, j_{0}\right)}^{*} \frac{y_{\left(l_{0}, i_{0}, j_{0}\right)}^{\prime}\left(t_{0}\right)}{y_{\left(l_{0}, i_{0}, j_{0}\right)}\left(t_{0}\right)}<\left[1-\psi_{l_{0}}^{*}\right] q_{i} \beta l_{0} \Theta\left(y^{*}\right)-y_{\left(l_{0}, i_{0}, j_{0}\right)}^{*}=0,
$$

or

$$
y_{\left(l_{0}, i_{0}, j_{0}\right)}^{*} \frac{y_{\left(l_{0}, i_{0}, j_{0}\right)}^{\prime}\left(t_{0}\right)}{y_{\left(l_{0}, i_{0}, j_{0}\right)}\left(t_{0}\right)}<-y_{\left(l_{0}, i_{0},(j-1)_{0}\right)}^{*}+y_{\left(l_{0}, i_{0}, j_{0}\right)}^{*}=0,
$$

and since $y_{\left(l_{0}, i_{0}, j_{0}\right)}^{*}>0$ and $y_{\left(l_{0}, i_{0}, j_{0}\right)}\left(t_{0}\right)>0$, we conclude that $y_{\left(l_{0}, i_{0}, j_{0}\right)}^{\prime}\left(t_{0}\right)<0$. Therefore, if $F\left(y\left(t_{0}\right)\right)>1,\left.F^{\prime}\right|_{(17)}\left(y\left(t_{0}\right)\right)<0$.

Similarly, we can testify if $F\left(y\left(t_{0}\right)\right)=1,\left.F^{\prime}\right|_{(17)}\left(y\left(t_{0}\right)\right) \leqslant 0$ and if $\left.f^{\prime}\right|_{(17)}\left(y\left(t_{0}\right)\right)>0$. If $f\left(y\left(t_{0}\right)\right)=1$, then $\left.f^{\prime}\right|_{(17)}\left(y\left(t_{0}\right)\right) \geqslant$ 0 . Denote

$$
\begin{aligned}
& U(x)=\max \{F(y)-1,0\}, \\
& V(y)=\max \{1-f(y), 0\} .
\end{aligned}
$$

Both $U(y)$ and $V(y)$ are continuous and nonnegative for $y \in \Delta_{\mathrm{N}}$. Notice that

$$
\left.U^{\prime}\right|_{(17)}(y(t)) \leqslant 0,\left.\quad V^{\prime}\right|_{(17)}(y(t)) \leqslant 0 .
$$

Letting $H_{U}=\left\{y \in \Delta_{\mathrm{N}}\left|U^{\prime}\right|_{(17)}(y(t))=0\right\}, H_{V}=\{y \in$ $\left.\Delta_{\mathrm{N}}\left|V^{\prime}\right|_{(17)}(y(t))=0\right\}$, then we have $H_{U}=\{y \mid 0 \leqslant$ $\left.y_{(l, i, j)} \leqslant y_{(l, i, j)}^{*}\right\}$ and $H_{V}=\left\{y \mid y_{(l, i, j)}^{*} \leqslant y_{(l, i, j)} \leqslant 1\right\} \cup\{0\}$. According to the LaSalle invariant set principle, any solution in $\Delta_{\mathrm{N}}$ will approach $H_{U} \cap H_{V}$. And $H_{U} \cap H_{V}=\left\{y^{*}\right\} \cup\{0\}$. 

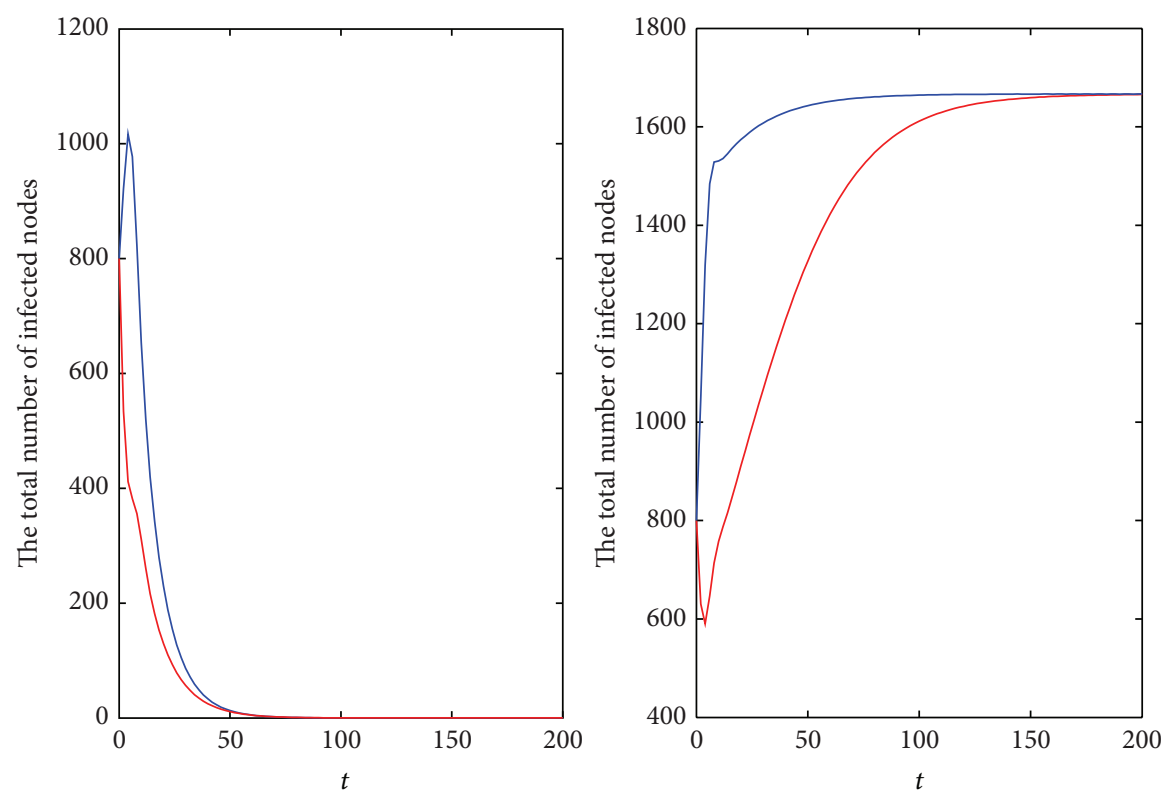

(a)
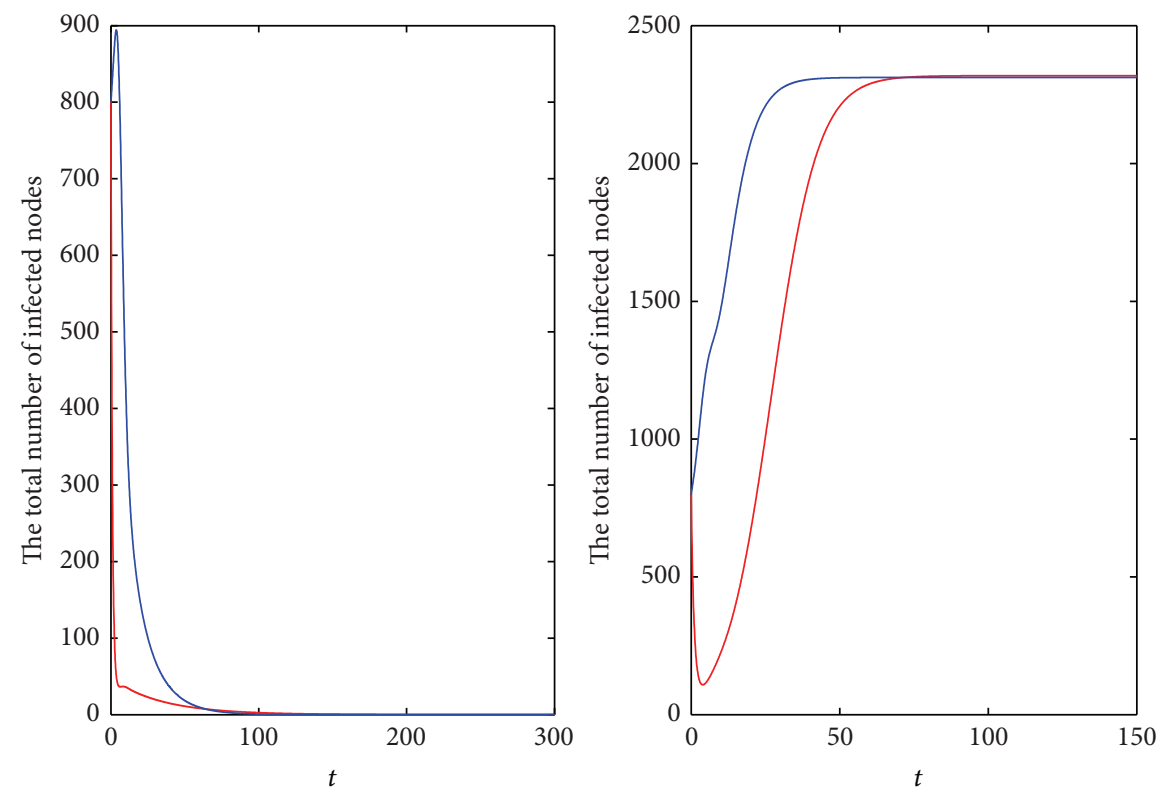

(b)

FIgURE 4: The consequences of different infectious period distributions on homogeneous (a) and BA scale-free (b) networks. In (a), $\beta=0.013$, $R_{0}=0.6$ (left plot) and $\beta=0.025, R_{0}=1.2$ (right plot). In (b), $\beta=0.013, R_{0}=0.87$ (left plot) and $\beta=0.025, R_{0}=1.66$ (right plot).

But if $y(t) \neq 0$, by Lemma 3 we know that $\liminf _{t \rightarrow \infty}\|y(t)\| \geqslant$ $m>0$. Then we conclude that any solution $y(t)$ of (17), such that $y(0) \in \Delta_{\mathrm{N}} \backslash\{\boldsymbol{0}\}$, satisfies $\lim _{t \rightarrow \infty} y(t)=y^{*}$, so $y=y^{*}$ is globally attractive in $\Delta_{N} \backslash\{\mathbf{0}\}$.

\section{Numerical Simulations and Sensitivity Analysis}

In this section, we first perform some sensitivity analysis of the basic reproduction number $R_{0}$ in terms of the model parameters on BA scale-free networks. Here $P(k)=$ $2 m^{2} k^{-v}(m=3, v=3),\langle k\rangle \approx 6, N=10^{4},\left\langle k^{2}\right\rangle \approx 50$.

From Figure 2, we can see the influence of transmission rate $\beta$ and the average infectious period $\langle i\rangle$. In particular, the influence of the diversity of the infectious periods is presented in Figure 2; the longer the infectious periods that the individuals have, the greater the basic reproduction number $R_{0}$ is. When $\beta$ is fixed, $R_{0}$ is a monotone increasing with the value of $\langle i\rangle$ and $R_{0}$ is a monotone increasing function of $\beta$ when $\langle i\rangle$ is fixed. At the same time, $R_{0}$ is linear function in terms of $\beta$ and $\langle i\rangle$. The basic reproduction number will 
increase with the increase of average infectious period and transmission rate.

We simulate the time series of total number of infected nodes on BA scale-free networks in Figure 3, which corresponds to $R_{0}<1$ and $R_{0}>1$, respectively. We can see that if $R_{0}<1$, the disease will disappear quickly; otherwise when $R_{0}>1$ the disease will persist in the system. So it is verified that $R_{0}$ is the threshold for the dynamics of disease.

In Figure 4, the blue line represents $M=8, q_{i}=0(i=$ $1,2, \ldots, 7)$, and $q_{8}=1$, and the red line represents $M=10$, $q_{1}=0.04, q_{2}=0.03, q_{3}=0.2, q_{10}=0.73$, and the rest of $q_{i}=0$. The mean of infectious periods for red or blue line is $\langle i\rangle=8$. Even though the final sizes for the same $R_{0}$ are the same, no matter $R_{0}>1$ or $R_{0}<1$, the cumulative numbers of infected individuals with different distributions are different.

\section{Conclusion and Discussion}

In this paper, we establish an SIS epidemic spreading model with an arbitrary distribution of infectious period and take network structure into consideration. The disease-free equilibrium is globally asymptotically stable when $R_{0}<1$. In the other case, there exists a unique endemic equilibrium such that it is globally attractive. Some numerical simulations are also performed to verify our theoretical results.

It is well known that, on a normal network, the basic reproduction number $R_{0}$ depends on the degree distribution, transmission rate, and recovery rate. However, when the characteristics of different nodes have a larger difference, the $R_{0}$ will depend on the distribution of these characteristics, such as the infectious period. From the equation of epidemic threshold, we have shown that the basic reproduction number $R_{0}$ depends on the heterogeneity of the social networks and the diversity of the infectious periods of the individuals. And we obtain that the heterogeneity of the network and the long infectious period resulting in the infection deteriorate into endemic more easily.

By modifying the staged progression model, we propose the multistaged progression model which contains several different gamma distributions. The linear combination of gamma distributions with different parameters can describe an arbitrarily distributed distribution of the infectious period. We find that the number of stable infected individuals for different infectious periods is the same; however, the cumulative numbers of the infected individuals are different corresponding to the different infectious period distributions. And numerical simulations show that different infectious period distributions can lead to different transmission processes. Hence our model can characterize the diversity of the infectious period during the disease transmission on complex networks more realistic.

\section{Conflict of Interests}

The authors declare that there is no conflict of interests regarding the publication of this paper.

\section{Acknowledgments}

This work is supported by the National Natural Science Foundation of China under Grant nos. 11331009, 11171314, 11147015, 11301490, 11301491, and 11101251, the Specialized Research Fund for the Doctoral Program of Higher Education (preferential development) no. 20121420130001, and the Youth Science Fund of Shanxi Province (2012021002-1).

\section{References}

[1] W. O. Kermack and A. G. McKendrick, "Contributions to the mathematical theory of epidemics," Proceedings of the Royal Society of London A, vol. 138, no. 834, pp. 55-83, 1932.

[2] R. M. Anderson and R. M. C. May, Infectious Diseases of Humans, Oxford University Press, Oxford, UK, 1991.

[3] J. D. Murray, Mathematical Biology I: An I ntroduction, Springer, New York, NY, USA, 2002.

[4] M. J. Keeling and P. Rohani, Modeling Infectious Diseases in Humans and Animals, Princeton University Press, Princeton, NJ, USA, 2008.

[5] H. J. Wearing, P. Rohani, and M. J. Keeling, "Appropriate models for the management of infectious diseases," PLoS Medicine, vol. 2, no. 7, Article ID e174, 2005.

[6] A. L. Lloyd, "Realistic distributions of infectious periods in epidemic models: changing patterns of persistence and dynamics," Theoretical Population Biology, vol. 60, no. 1, pp. 59-71, 2001.

[7] Z. Feng, D. Xu, and H. Zhao, "Epidemiological models with non-exponentially distributed disease stages and applications to disease control," Bulletin of Mathematical Biology, vol. 69, no. 5, pp. 1511-1536, 2007.

[8] S. H. Strogatz, "Exploring complex networks," Nature, vol. 410, no. 6825, pp. 268-276, 2001.

[9] R. Pastor-Satorras and A. Vespignani, "Epidemic spreading in scale-free networks," Physical Review Letters, vol. 86, no. 14, pp. 3200-3203, 2001.

[10] R. Yang, B.-H. Wang, J. Ren et al., "Epidemic spreading on heterogeneous networks with identical infectivity," Physics Letters A, vol. 364, no. 3-4, pp. 189-193, 2007.

[11] M. Barthélemy, A. Barrat, R. Pastor-Satorras, and A. Vespignani, "Velocity and hierarchical spread of epidemic outbreaks in scale-free networks," Physical Review Letters, vol. 92, no. 17, Article ID 178701, 2004.

[12] H. F. Zhang, M. Small, and X. C. Fu, "Staged progression model for epidemic spread on homogeneous and heterogeneous networks," Journal of Systems Science \& Complexity, vol. 24, no. 4, pp. 619-630, 2011.

[13] L. Zager and G. Verghese, "Epidemic thresholds for infections in uncertain networks," Complexity, vol. 14, no. 4, pp. 12-25, 2009.

[14] H. B. Guo and M. Y. Li, "Global dynamics of a staged progression model for infectious diseases," Mathematical Biosciences and Engineering, vol. 3, no. 3, pp. 513-525, 2006.

[15] L. M. Leemis, "Relationships among common univariate distributions," The American Statistician, vol. 40, no. 2, pp. 143-146, 1986.

[16] P. van den Driessche and J. Watmough, "Reproduction numbers and sub-threshold endemic equilibria for compartmental models of disease transmission," Mathematical Biosciences, vol. 180, no. 1, pp. 29-48, 2002.

[17] Y. Wang, Z. Jin, Z. Yang, Z. A. Zhang, and G. Sun, "Global analysis of an SIS model with an infective vector on complex 
networks," Nonlinear Analysis: Real World Applications, vol. 13, no. 2, pp. 543-557, 2012.

[18] J. A. Yorke, "Invariance for ordinary differential equations," Mathematical Systems Theory, vol. 1, no. 4, pp. 353-372, 1967.

[19] A. d'Onofrio, "A note on the global behaviour of the networkbased SIS epidemic model," Nonlinear Analysis: Real World Applications, vol. 9, no. 4, pp. 1567-1572, 2008.

[20] A. Lajmanovich and J. A. Yorke, "A deterministic model for gonorrhea in a nonhomogeneous population," Mathematical Biosciences, vol. 28, no. 3, pp. 221-236, 1976. 


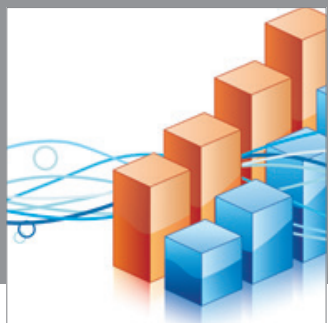

Advances in

Operations Research

mansans

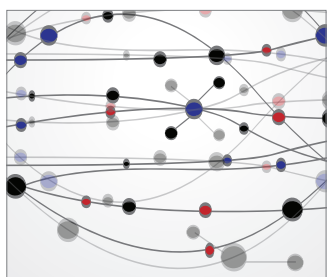

The Scientific World Journal
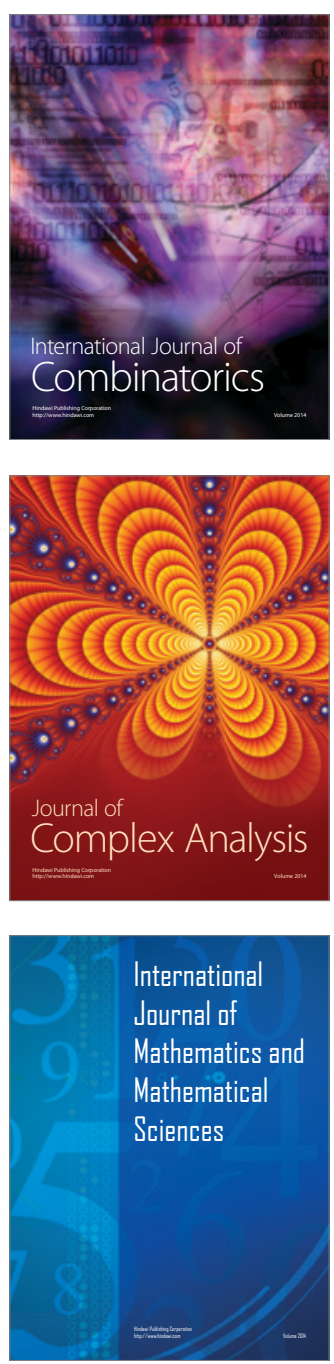
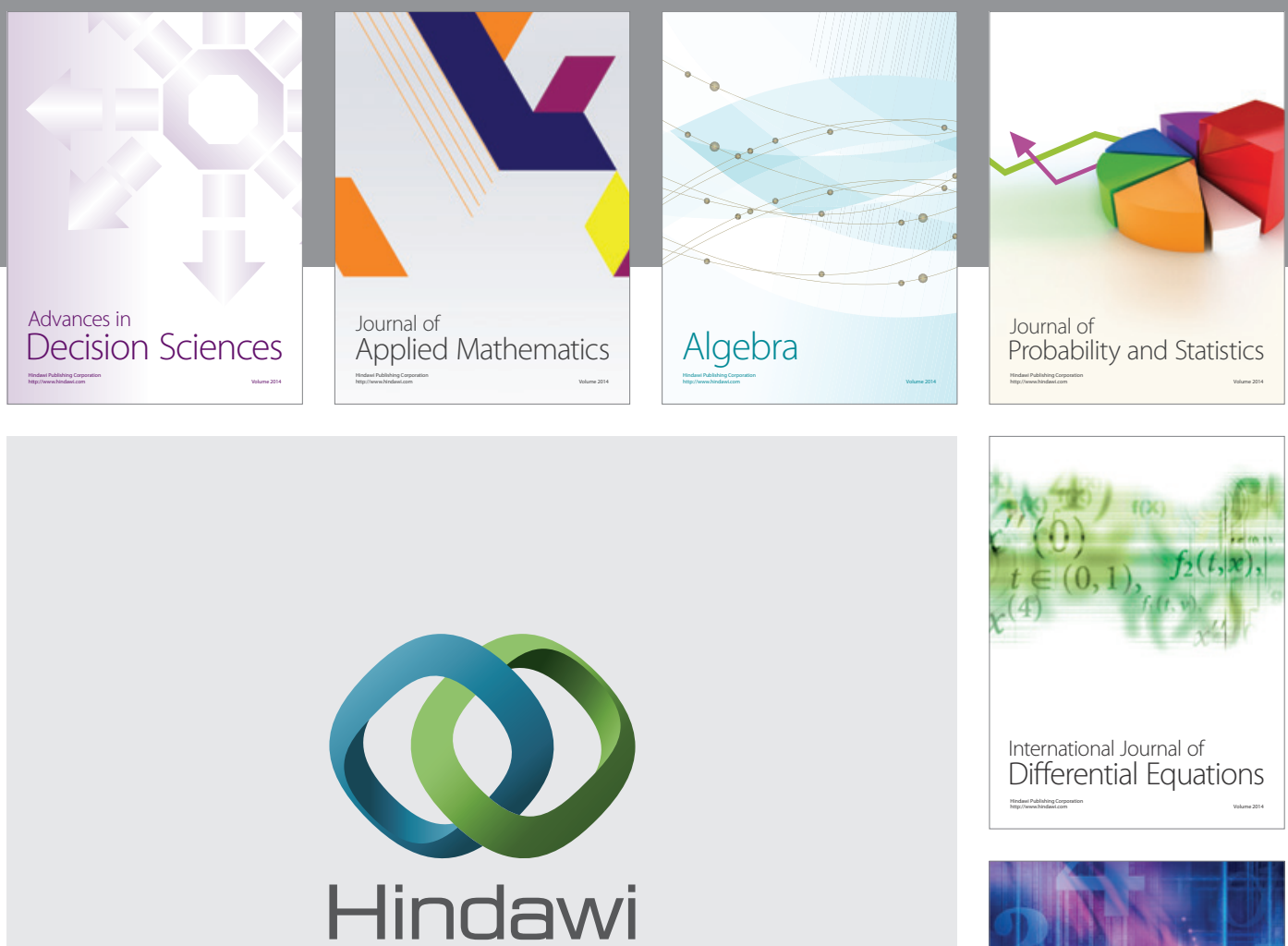

Submit your manuscripts at http://www.hindawi.com
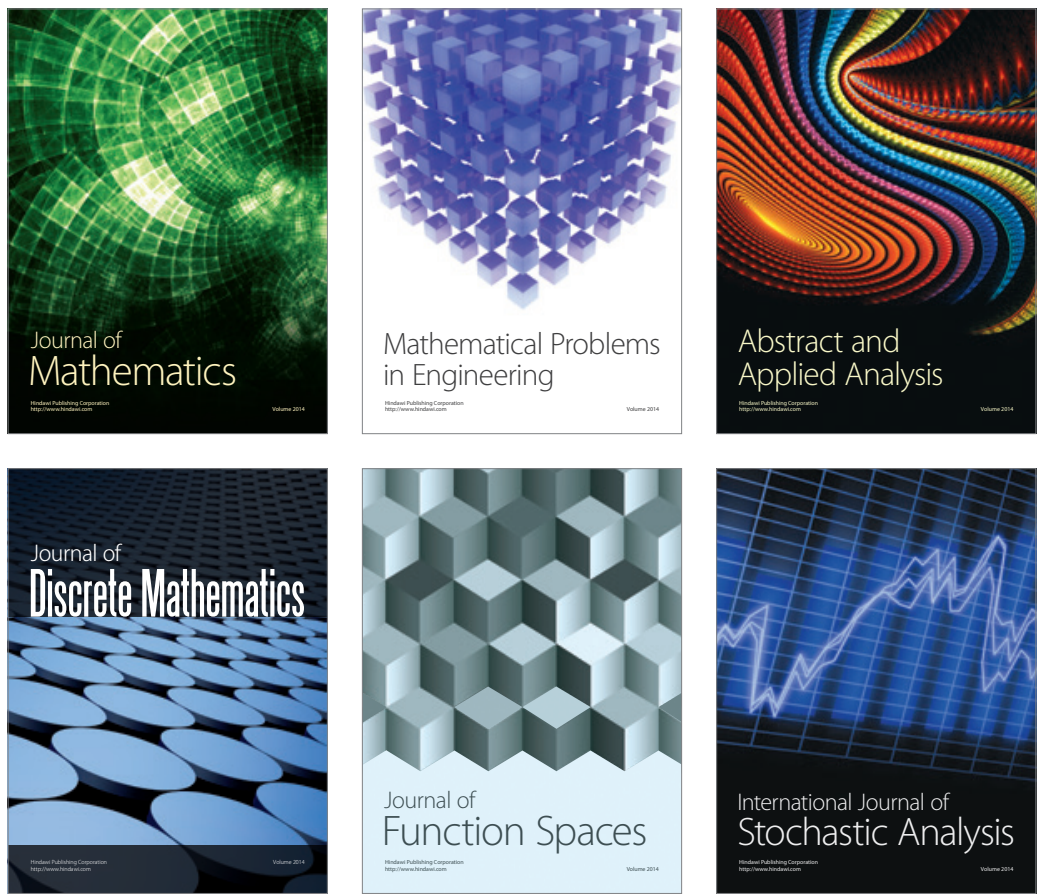

Journal of

Function Spaces

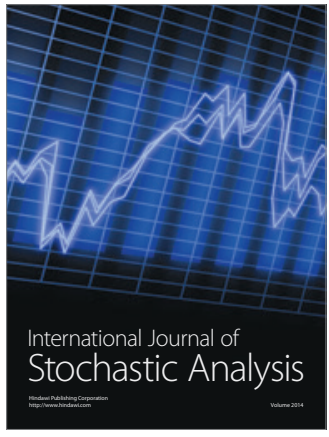

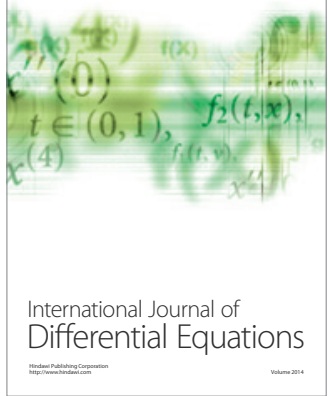
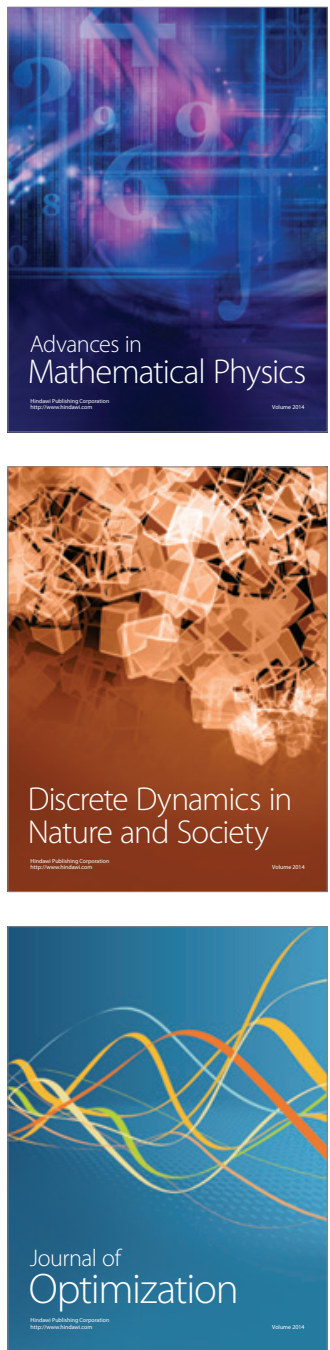